\title{
BIOTECNOLÓGÍCAS APLICADAS AL CULTIVO DEL BONIATO IPOMOEA BATATAS (L.)
LAM. Y SU RELACIÓN CON LA CONSERVACIÓN DE LA BIODIVERSIDAD
}

\section{Orlando S. González Panequel, María M. Hernández Espi- nosa ${ }^{2}$, Juan J. Silva Pupo ${ }^{1}$, Angel Espinosa Reyes ${ }^{1}$ y Carlos Ros Araluce ${ }^{1}$}

1 Centro de Estudios de Biotecnología Vegetal. Facultad de Ciencias Agrícolas. Universidad de Granma. Granma, Cuba.

opaneque@inca.edu.cu

2 Departamento de Genética y Mejoramiento de las Plantas. Instituto Nacional de Ciencias Agrícolas la Habana. Cuba.

\section{INTRODUCCIÓN}

El desarrollo del cultivo del boniato (Ipomoea batatas (L.) Lam.) ofrece diversas ventajas económicas para muchos países, ya que se puede emplear en la alimentación humana y animal, así como en la industria y puede también ser utilizado como forraje y abono verde. El área de siembra a nivel mundial es de aproximadamente 8.618.866 ha, con una producción de 127.139.553 t en el año. El 90,5\% del área dedicada al cultivo se encuentra en el continente asiático, donde se obtiene el $91 \%$ de la producción mundial (FAO, 2004).
Actualmente, se exige en la producción agrícola una mayor productividad con criterios de sostenibilidad y la biotecnología tiene una importante función en el logro de estas metas, ya que los nuevos productos biotecnológicos deben ofrecer apoyo a los programas de propagación acelerada para evitar los posibles efectos en el medio ambiente. El empleo de nuevos métodos ha propiciado avances más rápidos en la obtención y multiplicación de genotipos.

Las tendencias mundiales abren un espacio cada vez mayor al uso de la biotecnología por contribuir ésta al aumento y mejora de la producción de alimentos (Sasson, 2001) en un momento en el que la humanidad se 
enfrenta al reto de cómo satisfacer las necesidades de una población creciente (Nichterlein, 2000).

En la actualidad, la propagación de plantas in vitro (Figura 1) a escala comercial se ha consolidado mundialmente y esto posibilita la multiplicación acelerada en muchas especies (Pérez, 1998) y permite obtener volúmenes de producción superiores en menos tiempo (Posada et al., 2003).

En trabajos realizados en el cultivo del boniato por López et al. (2002), se expone que la impor-

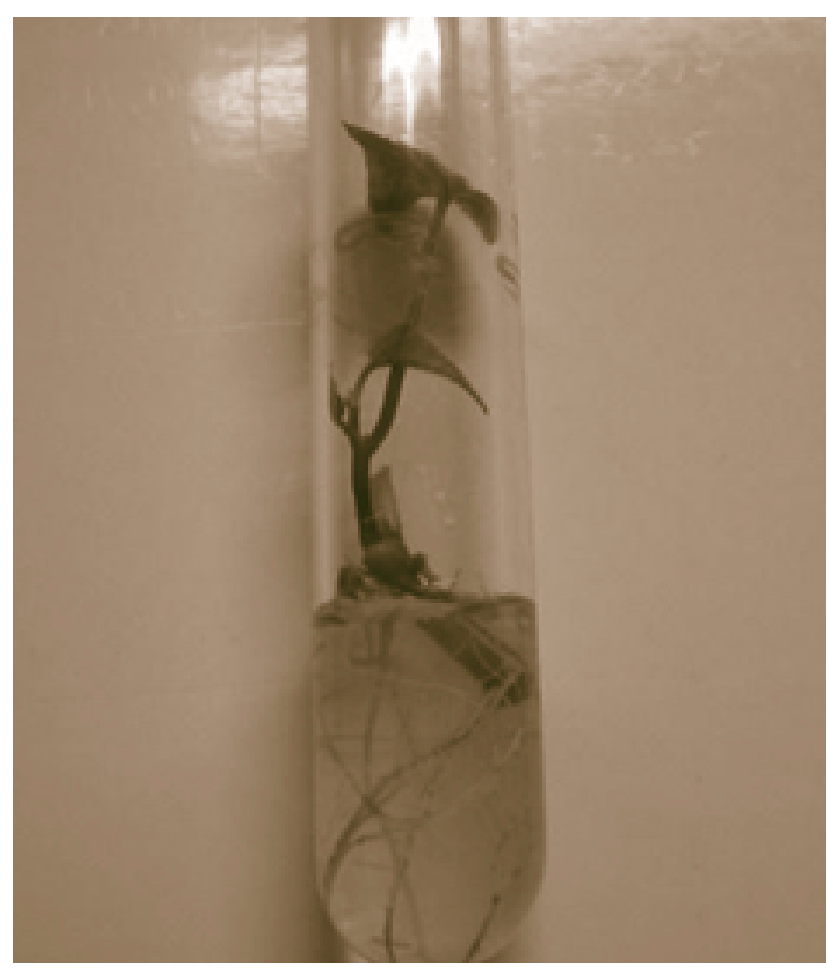

Figura 1. Cultivo in vitro del boniato.

tancia del cultivo viene dada por su hábito de consumo y su ciclo vegetativo corto, siendo una de las alternativas para la propagación masiva de esta especie, el uso de las técnicas de cultivo de tejidos, que permiten una propagación clonal rápida en un período breve de tiempo.

Actualmente se hacen necesarios estudios para evitar la disminución de la diversidad biológica, lo que produce un daño irreparable en la naturaleza. De ahí la necesidad de desarrollar acciones compatibles con la conservación y el uso sostenible de la diversidad biológica como única garantía de preservar el patrimonio natural de la vida en el planeta (Universidad para todos, 2003).
Teniendo en consideración que el cultivo del boniato constituye uno de los de mayor extensión y consumo a nivel mundial, se hace necesario dar a conocer aspectos relacionados con su cultivo y su relación con la conservación de la biodiversidad mediante la aplicación de técnicas de cultivo de tejidos vegetales del mismo.

\section{ORIGEN, CARACTERÍSTICAS E IMPOR- TANCIA ECONOMICA DEL BONIATO}

Existen dos teorías sobre el origen del boniato, una lo considera oriundo del continente americano, situándolo en México y Centroamérica, en virtud de la división del material genético y por la evidencia arqueológica de la antigüedad de su cultivo. La otra teoría defiende su origen en China. Sin embargo, en la actualidad se acepta que es originario de América, al tener en cuenta que existe mayor cantidad de especies que en el continente Asiático, con alto potencial de rendimiento y con mayor desarrollo vegetativo, favoreciendo la floración y la fructificación.

Es uno de los cultivos tradicionales más antiguos y valiosos, sembrados ampliamente en los países en vías de desarrollo. Destaca por su rusticidad y su alta productividad por unidad de área y de tiempo y es un cultivo rico en vitaminas y minerales. En muchos países su principal uso está dado en la alimentación humana, porcinos, bovinos, aves, ovinos y conejos.

Es una Convolvulácea que responde a la división Macrophyllophyta, clase Magnoliatae, subdivisión Magnoliophytina y orden Polemoniales. Es un hexaploide natural $(2 \mathrm{n}=6 \mathrm{x}=90)$, a diferencia de la mayor parte de los miembros del género que son diploides $(2 \mathrm{n}=30)$ o tetraploides.

Es una planta anual, herbácea, rastrera, en ocasiones con ápices volubles, glabra o pubescente, con raíces adventicias y tuberosas (Figura 2). Se propaga vegetativamente por segmentos de tallos y raramente por las raíces tuberosas y semillas.

El género Ipomoea tiene más de 400 especies y desde el punto de vista económico, la única especie cultivada con destino a la alimentación es Ipomoea batatas. Según Mortley (1993), no se conocen efectos de toxicidad en lo referente a este cultivo.

La especie Ipomoea batatas está conformada por un alto número de clones que difieren en valor alimenti- 


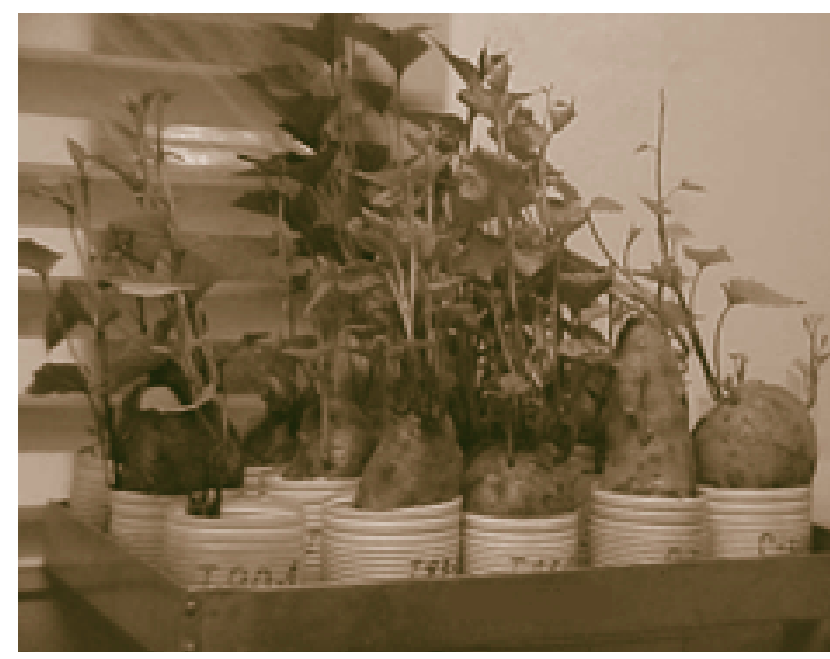

Figura 2. Tubérculos de boniato para experimentación.

cio, tamaño, forma, color de las raíces tuberosas, período de maduración, producción y resistencia a plagas y enfermedades, y en los últimos años se ha aumentado la producción agrícola de clones comerciales de elevado potencial de rendimiento.

\section{IMPACTO EN LA BIODIVERSIDAD Y EN LA ALIMENTACIÓN HUMANA}

Es de todos conocido la influencia que ejerce el clima sobre el crecimiento y la producción de los cultivos, que en casi la totalidad de ellos determina su distribución en las distintas regiones del planeta (Soto y Morales, 1996).

El actual desarrollo de la agricultura ha traído consigo la propagación de nuevos cultivares comerciales de altos potenciales de rendimiento, los cuales han ido desplazando a los tradicionalmente utilizados. Se han desarrollado evaluaciones y regionalizaciones de clones de boniato en diferentes partes del mundo para la propagación de nuevos clones de interés comercial, ya sea con el empleo de las técnicas tradicionales de siembra o con el empleo de las técnicas de cultivo de tejidos vegetales para la producción de material vegetal.

La mayor parte de los esquemas de multiplicación utilizados en el boniato han estado basados, fundamentalmente, en la multiplicación agámica por la vía tradicional de los clones existentes y la introducción de otros foráneos, contándose actualmente con un gran número de clones. Asimismo, han sido realizadas investigaciones que apoyan y complementan los pro- gramas de establecimiento de variedades clonales por estas vías. No obstante, existen aún aspectos en los que se requiere un mayor esfuerzo y profundización en las investigaciones, fundamentalmente, las encaminadas a la multiplicación de nuevos clones con el empleo de las técnicas de avanzadas de cultivo de tejidos vegetales.

Según Cornide (2002), se impone la búsqueda de alternativas a los productos agrícolas de cultivos con alta capacidad instalada, que ayuden a afrontar las caídas del valor de cambio y la inestabilidad del mercado internacional.

Es bueno señalar que a nivel mundial se trabaja en el cultivo del boniato para incrementar la producción, adaptación a diferentes regiones y resistencia a plagas y enfermedades, entre los que podemos citar los resultados alcanzados por el Centro Internacional de la Papa (CIP) Perú, y en Cuba se han logrado magníficos resultados en investigaciones realizadas en éste cultivo en diferentes centros, entre los que se destaca el Instituto Nacional de Investigaciones de Viandas Tropicales (INIVIT), Santo Domingo, Villa Clara, en lo referente a la obtención y multiplicación de nuevos clones.

Cada día se trabaja en los programas de multiplicación de nuevos clones que presentan una mejor adaptación a las diversas regiones con altos rendimientos. La tendencia que existe en el ámbito mundial, en lo referente al cultivo del boniato, es la multiplicación de clones precoces de alta productividad de color amarillo, ricos en b- carotenos, que se adapten a las condiciones zonales de cada región, mejorar la calidad de la semilla y lograr resistencia a plagas y enfermedades.

\section{EL CULTIVO DE TEJIDOS EN LA MICRO- PROPAGACIÓN DE ESPECIES VEGETALES}

El desarrollo alcanzado por las técnicas de cultivo de tejidos vegetales, desde sus inicios hasta la fecha, ha dado un gran aporte a la agricultura y en la actualidad constituye una vía fundamental en la actividad científico tecnológica (Cevallos, 2000).

En el cultivo del boniato se hace necesario la búsqueda de nuevas técnicas para la multiplicación acelerada del mismo como una vía alternativa, ya que la vía de propagación tradicional es por "semilla agámica" y esto trae consigo dificultades en el empleo del mismo en sucesivas generaciones y la reproducción por semilla botánica es poco común. 
Según Sasson (2001), los métodos biotecnológicos aplicados a las plantas, conducen a una racionalización de los trabajos de multiplicación que permiten realizarse por los métodos tradicionales y posibilitan la multiplicación de las especies en grandes cantidades y en corto tiempo. Hoy en día es muy importante la aplicación de las técnicas de cultivo in vitro para la propagación de especies vegetales en el cual se agrupan diversos aspectos relacionados con el cultivo, genéticos y biológicos; cuya aplicación ha logrado elevar el rendimiento y disminuir considerablemente las pérdidas.

La biotecnología ofrece grandes posibilidades en la multiplicación de especies vegetales, pero se hace necesario conocer las características de las poblaciones a multiplicar. Con el surgimiento de las técnicas de cultivo in vitro, se abre una amplia perspectiva para su empleo en la micropropagación de especies vegetales de interés. El cultivo de tejidos vegetales es una alternativa para la producción de semillas y juega un rol importante en el suministro de semillas de alta calidad, aportando una esencial contribución a la propagación acelerada de los cultivos.

En los últimos años, las técnicas de cultivo de tejidos vegetales han sobrepasado la fase de investigación, esto ha traído como consecuencia el establecimiento de laboratorios que producen y propagan masivamente muchas especies a escala comercial (Jiménez, 1998). Según Borroto (2002), la propagación in vitro tiene muchas ventajas, en especial la de una mayor tasa de multiplicación de material libre de plagas y enfermedades en un pequeño espacio que permite la producción de forma rápida y económica de cantidades muy grandes de plantas de alta calidad.

Las técnicas de cultivo de tejidos vegetales están establecidas en algunos cultivos para la propagación clonal de genotipos selectos. Esta importante función del cultivo de tejidos ha aumentado con los adelantos que se han producido durante los últimos años en los métodos para la erradicación de organismos patógenos, micropropagación, manipulación genética, conservación y distribución de germoplasma (Alvarado et al., 2003).

La siembra de las vitroplantas en condiciones naturales constituye una etapa importante y según Vega et al. (2000), la fase final de aclimatización de las vitroplantas es trascendental para la propagación, pues del resultado de ésta dependerá en gran medida la calidad final de las plantas y la eficiencia total del proceso.
Agramonte et al. (1998), plantearon que la aclimatización de las vitroplantas en condiciones ambientales, constituye una etapa muy crítica en la propagación masiva mediante el cultivo de tejidos vegetales, ya que se exponen a un estrés cuando son colocadas en las condiciones del ambiente.

Las plántulas obtenidas in vitro son sensibles a los cambios que puedan ocurrir en su medio, por lo que no es posible trasladarlas del laboratorio al suelo de forma directa y se requiere de un tiempo de adaptación en condiciones que les permitan alcanzar un crecimiento y vigor para ser plantadas definitivamente en el suelo (Granada, 1990).

\section{CONCLUSIONES}

El hombre, a lo largo de los siglos de existencia sobre la tierra, ha usado los recursos naturales para satisfacer sus necesidades materiales y espirituales y cuando esta acción se convierte en explotación irracional, sin armonía con la dinámica de los ecosistemas, trae consigo la desaparición de los recursos e impide de manera insoslayable el logro del desarrollo sostenible. La pérdida de recursos biológicos y de diversidad, pone en peligro el suministro de alimentos y es por ello que, cada vez adquiere mayor urgencia la necesidad de salvaguardar estos recursos.

El estudio de los cultivos más comúnmente consumidos por la población es de vital importancia para fortalecer la producción y calidad de los mismos mediante métodos compatibles con la conservación del medio ambiente. En este sentido el uso de los avances biotecnológicos marcan nuevas pautas en la investigación y en la búsqueda de nuevas vías en los programas de propagación acelerada de los cultivos de interés y constituyen una herramienta más de vital importancia en el desarrollo de protocolos de trabajo de investigación en estos cultivos.

\section{BIBLIOGRAFÍA}

AGRAMONTE, D.; D. RAMÍREZ; M. PÉREZ; O. GUTIÉRREZ; J. PÉREZ Y M. NUÑEZ. (1998). Manejo integrado de vitroplantas en la fase de aclimatización. Programa y Resúmenes. XI Seminario Científico del INCA. Cultivos Tropicales. La Habana. 115 p. 
ALVARADO, Y.; M. CRUZ; N. PORTAL; L. GARCÍA; M. FREIRE; E. QUIALA Y R. GÓMEZ. (2003). Estrategia de trabajo para el control de la contaminación bacteriana en la micropropagación de la caña de azúcar. Programa y Resúmenes. Taller Internacional de Biotecnología Vegetal (BioVeg 2003). Centro de Bioplantas. CD-ROM. Ciego de Ávila. Cuba.

BORROTO, C. (2002). Biotecnología Agropecuaria. Cuba. Amanecer del Tercer Milenio. Ciencia, Sociedad y Tecnología. Editorial DEBATE. La Habana. 414 p.

CEVALLOS, M. (2000). Establecimiento de una metodología eficiente en el proceso de embriogénesis somática del cafeto (Coffea sp.), mediante el uso de marcadores morfohistológicos y moleculares. Tesis de Grado (Dr. en Ciencias Agrícolas). INCA. La Habana. 136 p.

CORNIDE, M.T. (2002). Genética Vegetal. Cuba. Amanecer del Tercer Milenio. Ciencia, Sociedad y Tecnología. Editorial DEBATE. La Habana. 414 p.

FAO. (2004). FAO Stat-Agricultse. Disponible en: http://www.fao/waicent/portal/statistces.asp.Visitada en fecha: 21 de Julio del 2005.

GRANADA, C.L. (1990). Manejo de plantas en invernadero. En: V.M. Villalobos (ed). Fundamentos Teórico-Práctico del Cultivo de Tejidos Vegetales. FAO (105). P. 85-88.

JIMÉNEZ, G.E. (1998). Generalidades del cultivo in vitro. En: J. Pérez (ed). Propagación y Mejora de Plantas por Biotecnología. Cap. 1. Vol. 1. IBP. Santa Clara. Cuba, P. 13-24.

LÓPEZ, J.; M. TORRES; C. BORROTO; R. TRUJILLO; M. DAQUINTA; R. GÓMEZ; M. GARCÍA; V. MEDERO; J. VENTURA; N. MONTANO; A. MORALES; M. CABRERA; A. ROBAINA; A. RAYAS Y C. PONS. (2002). Metodología para la propagación in vitro del boniato. Programa y Resúmenes. Jornada XXXV Aniversario del INIVIT. Santo Domingo. Villa Clara. Cuba. P. 18.

MORTLEY, C. (1993). Manganese toxicity and tolerance in Sweet Potato. Hort Sciences Soil Nutrition and Fertilizer, 28(8): 812-813.

NICHTERLEIN, K. (2000). Workshop on mutation and in vitro culture techniques for the improvement of vegetatively propagated tropical food crop.
Curso de Investigaciones Agronómicas. Universidad de Costa Rica. P. 56.

PÉREZ, J.P. (1998). Propagación y Mejora Genética de Plantas por Biotecnología. Vol. 1. IBP. Villa Clara. $390 \mathrm{p}$.

POSADA, L.; R. GÓMEZ; M. REYES Y L. ALVAREZ. (2003). Empleo de los sistemas de inmersión temporal (RITA) en la propagación de plantas vía organogénesis en caña de azúcar y bananos. Biotecnología Vegetal, 3(1): 3-8.

SASSON, A. (2001). Cultivos Transgénicos: Hechos y Desafíos. Editorial ELFOS Scientia. La Habana. $377 \mathrm{p}$.

SOTO, F. Y D. MORALES. (1996). Relación de la temperatura del aire, la humedad relativa y la radiación global con el crecimiento de plántulas de cafeto. Cultivos Tropicales, 17(3): 41-44.

UNIVERSIDAD PARA TODOS. (2003). Cuaderno de Diversidad Biológica. Tabloide. La Habana. $31 \mathrm{p}$.

VEGA, R.; L.E. RODRÍGUEZ; J.J. SAGUE Y Y. SANTIAGO. (2000). Utilización de substratos alternativos en la aclimatización de vitroplantas de plátano cv. FHIA 01. XII Seminario Científico del INCA. Programa y Resúmenes. La Habana. P. 188.

\section{AGRADECIMIENTOS}

Agradecemos profundamente a los investigadores: Dr. Sergio Rodríguez y al Dr. Alfredo Morales Tejón, pertenecientes al Instituto Nacional de Investigaciones de Viandas Tropicales (INIVIT), Santo Domingo, Villa Clara, Cuba. A la Dra. Silvia Montes Cruz y Mirtha López Machado, pertenecientes al Instituto Nacional de Ciencias Agrícolas de la Habana (INCA), por las sugerencias y ayuda brindada en los trabajos de estudios realizados en el presente cultivo y la ejecución de éste trabajo. Además, deseamos hacer patentes los más profundos agradecimientos a la "DIPUTACIÓN FORAL de BISKAIA" y a la ONG's "ASOCIACIÓN de EUSKADI-CUBA" por la ayuda brindada en el financiamiento del Proyecto titulado: "Apoyo al Desarrollo de la Biotecnología Vegetal y la Agricultura Urbana en la Provincia Granma”, lo cual se encuentra estrechamente relacionado con lo que en éste trabajo se expone y los resultados alcanzados en biotecnología vegetal en la Universidad de Granma. 